\title{
A NOVEL INLET MODEL FOR TWO-PHASE BUBBLY AXIAL FLOW THROUGH A TUBE BUNDLE
}

\author{
Laurent De Moerloose $^{*, 1}$, Michel De Paepe Pa $^{1,2}$ and Joris Degroote ${ }^{1,2}$ \\ * Corresponding author. E-mail: laurent.demoerloose@ugent.be \\ ${ }^{1}$ Department of Electromechanical, Systems and Metal Engineering, Ghent University, \\ Sint-Pietersnieuwstraat 41-B4, 9000 Ghent, Belgium. \\ ${ }^{2}$ FlandersMake@UGent corelab EEDT MP
}

Key words: computational fluid dynamics, two-phase flow, flow-induced vibrations, tube bundle

\begin{abstract}
In numerical research about two-phase flow in tube bundles, mainly two trends are distinguishable. On the one hand, some studies start from the assumption that only relatively small bubbles occur in a continuous liquid flow, for which typically Eulerian-Lagrangian modelling is appropriate. This approach is not suitable when modelling flow-induced vibrations in tube bundles, as the most severe vibration conditions are achieved in intermittent, churn or slug flow. On the other hand, some studies focus on accurate numerical modelling of the bubble shape. In that case, it is customary to start from a bubble which is already present inside the domain. However, in order to simulate a sufficiently long flow time which is of interest in the current research, the bubbles should enter the domain through an inlet boundary. In this paper, a new inlet model is proposed which defines a transient inlet boundary condition to be applied in a subsequent Eulerian simulation, more specifically using the Volume-Of-Fluid method.

In the first part of this paper, the inlet model is described. The model guarantees the introduction of a userspecified amount of gas during a set time-interval at locations in space and time that are chosen randomly. Subsequently, the model is tested on a $3 \times 5$ tube bundle subjected to an axially flowing air/water mixture. The computational time required to complete the inlet model is reported for different values of the most important model parameters. Finally, the computational effort of the new inlet model is compared to that of a simulation with a precursor-domain where bubbles are created by break-up of air jets imposed at the inlet.
\end{abstract}

\section{INTRODUCTION}

Tube bundles subjected to axial flow have been the topic of an extensive number of experimental studies, especially with the advent of Pressurized Water Reactors (PWR) in nuclear power plants. Today, similar tube bundle geometries are still under investigation in both numerical $[3,4]$ and experimental studies $[5,6]$, often using water or air as working fluid; this is referred to as single-phase flow. As a result, the single-phase parallel flow through a tube bundle is relatively well understood. Meanwhile, some experimental papers about two-phase flow, i.e. a mixture of gas and liquid, have been published, often aimed at analyzing the altered vibration behaviour compared to single-phase flow [7] in heat exchangers, 
e.g. in the Boiling Water Reactors (BWR). With the advent of computational power, numerical modelling of two-phase flow has gained importance in the last two decades.

On the one hand, some numerical studies start from the assumption that only relatively small bubbles occur in a continuous liquid flow, for which typically Eulerian-Lagrangian modelling [8] is appropriate. This approach is not suitable when modelling flow-induced vibrations in tube bundles, as the most severe vibration conditions are achieved in intermittent, churn or slug flow. On the other hand, some studies focus on accurate numerical modelling of the bubble shape. In that case, it is customary to start from a bubble which is already present inside the domain.

However, with respect to tube bundles specifically, these two approaches are less useful. The vibrations occurring in tube bundles are mostly correlated to the presence of large liquid slugs and/or air bubbles [9]. For such large structures, accurate modelling of the interface below the cell size is not required and therefore encompasses an unnecessary computational cost. On the other hand, in the EulerianLagrangian approach, bubble interaction, impact or shape change should be modelled explicitly, e.g. based on coalescence criteria [11], but this is only done in a limited number of studies. One example is the work by Trapp et al. [14], who already successfully applied an Eulerian-Lagrangian framework to calculate bubble growth and changing flow topology, but the pressure drop, bubble velocity and forces on the surrounding or immersed tubes have not been validated. Moreover, a Lagrangian model for the bubbles is closed with semi-empirical models for lift and drag forces [10] which are typically not sufficiently accurate for large air structures such as encountered in slug or intermittent flow. Some attempts have been made to increase the accuracy of such closure models in an Eulerian-Lagrangian

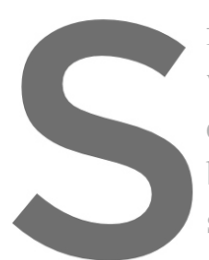
framework [12, 13], but whereas non-spherical bubt coalescence and growth bubble forces, at least wit structure. This leads to the called one-fluid models in this particular case.
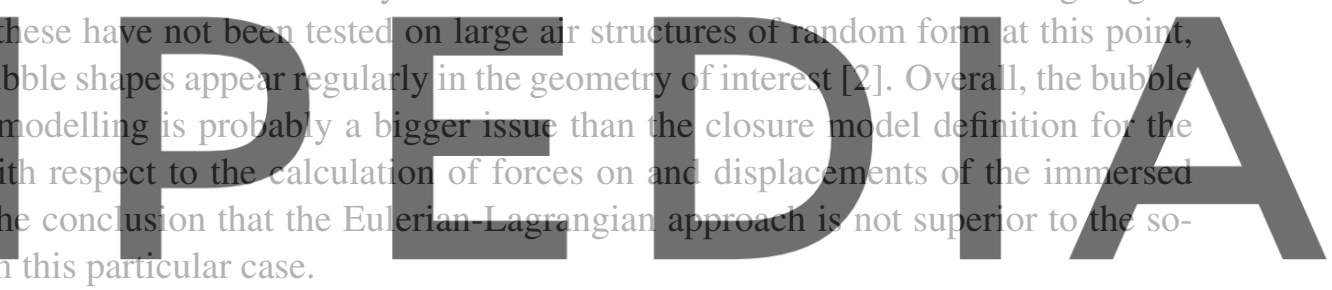

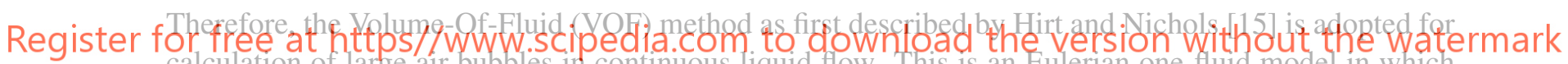
calculation of large air bubbles in continuous liquid How. This is an Eulerian one-fluid model in

$$
\rho_{m}=\alpha_{w} \rho_{w}+\left(1-\alpha_{w}\right) \rho_{a}
$$

and

$$
\mu_{m}=\alpha_{w} \mu_{w}+\left(1-\alpha_{w}\right) \mu_{a}
$$

where the indices $w$ and $a$ denote the properties of water and air, respectively. The variable $\alpha_{w}$ is defined as the volume fraction of water present in a specific cell. In order to close the one-fluid model, a scalar transport equation for $\alpha_{w}$ is solved. In the open-source, finite volume package OpenFOAM ${ }^{\circledR} 4.1$, the scalar transport equation is the following (where $U$ is the flow velocity):

$$
\frac{\partial \alpha_{w}}{\partial t}+\nabla \cdot\left(\alpha_{w} \vec{U}\right)+\nabla \cdot\left[\alpha_{w}\left(1-\alpha_{w}\right) \vec{U}_{l g}\right]=0 .
$$

The VOF method has proven to be useful in two-phase flow simulations where the two phases are divided by an interface stretching over multiple cells. This is also the case for liquid flows containing large air 
bubbles such as in the slug or intermittent flow regime which are of particular interest in the present research [16]. The same method has been succesfully applied to internal slug flow in previous work [17].

In the current research, the aim is to investigate the forces on tube bundles subjected to a water flow containing multiple large air bubbles over a longer period of time. In two-phase slug or intermittent flow, this necessitates a transient inlet which is able to define bubbles of random size at a random location and time instant. To the authors' knowledge, no method described in literature efficiently generates large bubbles with a random distribution in size and location. In this paper, a new model is proposed which defines a transient inlet boundary condition to be applied in a subsequent Computational Fluid Dynamics (CFD) simulation. This model allows the definition of spherical bubbles of random size at a random time instant and at a random location on the inlet face. Firstly, the algorithm of said model will be discussed in Section 2. How long the model takes to complete and how this duration scales with respect to the model's most important input parameters, is analyzed in Section 3. Finally, the subsequent simulation using the modified inlet condition will be compared to two precursor domain calculations in Section 4 . The results presented in this paper have been obtained for a tube bundle geometry containing 15 full cylinders organised in a square array. The tube diameter and array pitch match the experiments of Ren et al. [1] and Liu et al. [2].

\section{INLET MODEL DESCRIPTION}

In this work, a new model is presented which defines such a transient inlet condition for an arbitrarily long time duration (set by the user). The unser sets the total flow time to be modelled (by getting a ste $t_{\text {start }}$ and end time $\left.t_{\text {end }}\right)$. $t_{\text {unit }} . U$ is the flow velocity smallest bubble to be moc amount of gas to be introduced, the parameter $m_{g}$ qu interval tunit, up to a user-defined tolerance $\varepsilon_{m g}$. T
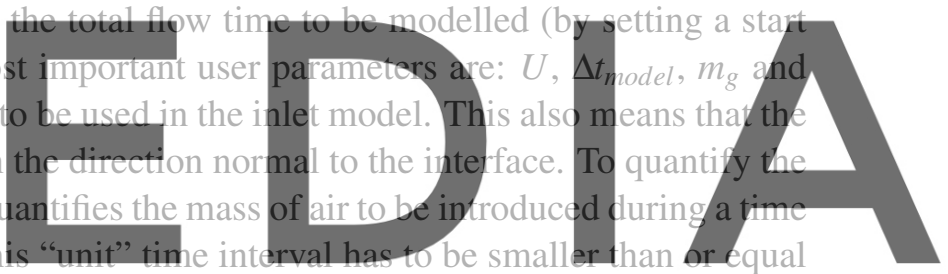

to the total flow time to be modelled and the ratio between both has to be an integer. The variable $t_{\text {unit }}$ can

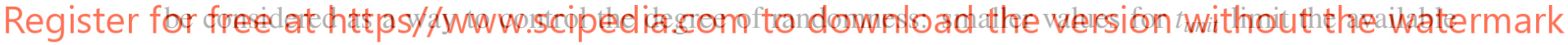
number of locations where a bubble can exist and therefore it is more likely that a more homogeneous distribution of bubbles is achieved, while large values allow the occurrence of zones with large or small concentrations of air.

Algorithm 1 shows the principle behind the inlet model. Slip between phases is not taken into account and therefore the air and water enter the domain at the same speed and moving in the direction of the inlet face normal. Hence, only the $\alpha_{w}$-profile is adapted in Algorithm 1. The variable $\alpha_{w}$ at spatial location corresponding to face with ID $i$ and in timestep $j$ is stored in matrix element $A_{i, j}$; consequently, $A \in \mathbb{R}^{N_{\text {faces }} x N_{\text {time }}}$. Index $i$ represents the unique ID given to each face located at the inlet and ranges from 0 till the number of cells at the inlet $N_{\text {faces }}$ minus one. For a face $i$, the face area is denoted with $S_{i}$. $N_{\text {time }}$ denotes the total number of time steps to be modelled and is equal to $\left(t_{\text {end }}-t_{\text {start }}\right) / \Delta t_{\text {model }}$. As such, each element $A_{i, j}$ can be interpreted as the $\alpha_{\text {water }}$-value in a virtual cell $C_{i, j}$, which is constructed from the inlet face $i$ and has a length $U . \Delta t_{\text {model }}$. Initially, all elements $A_{i, j}$ are set to one, after which air bubbles are introduced at specific locations in time and space by setting $A_{i, j}=0$ in the corresponding matrix locations. The inserted bubbles have a random size - denoted by the variable $m$ - and location, but their shape is predefined; each bubble shape receives an index $b$. Overall, a bubble is defined by four 
parameters: the location of the bubble center in space $\left(i_{c}\right)$ and in time $\left(j_{c}\right)$, the bubble shape $(b)$ and the bubble size $(m)$. Each of these variables are chosen randomly at the start a new iteration. The bubble size $m$ is located between 0 and the still available amount of gas $m_{g}-m_{d e f}$, where $m_{d e f}$ is a variable which keeps track of the mass of gas already introduced in previous iterations.

It is clear that Algorithm 1 randomly selects the bubble shape, size and location, after which it is checked for each possible location in space $i$ and time $j$ whether the cell center $C_{i, j}$ falls inside the new bubble. The user can decide whether a newly-defined bubble can intersect with a previously introduced bubble (which is checked on line 1.24) and whether a bubble can intersect with a wall (which is checked on line 1.33). Finally, if the newly-defined bubble complies to all the requirements, the matrix $A$ is updated and the amount of gas that was introduced during this particular time interval $t_{\text {unit }}$ is updated in the parameter $m_{d e f}$. If one or more criteria are not met, the iteration is stopped and new bubble parameters $\left(i_{c}, j_{c}, b, m\right)$ are defined in a subsequent iteration. Clearly, choosing an excessively high $m_{g}$ or low $t_{\text {unit }}$ may lead to insufficient space to define all required bubbles and would therefore put the inlet model into an infinite loop. Hence, a counter $N_{\text {fail }}$ keeps track of the number of consecutive failed iterations. If this counter reaches a certain hard-coded value, i.e. 1000, the operation is stopped and the user is prompted by the programme to review the specified settings.

The model was defined in Python and was coupled to the software package OpenFOAM ${ }^{\circledR}$ 4.1. Nevertheless, the solver-specific part of the code is limited to reading the coordinates and face areas of the inlet boundary on the one hand and the final conversion of the newly-defined boundary condition to a format readable by OpenFOAM ${ }^{\circledR}$ on the other hand. The latter is done by using the timeVaryingMappedFixed-

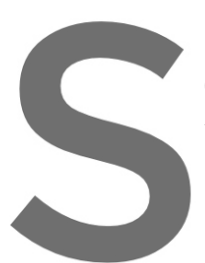
Value-boundary condition in OpenFOAM ${ }^{\mathbb{R}}$. Contrariwise, the actual definition of the in let bounday condition - such as shown in is independent of the flow solver and can be used in any flow solver using the VOF-method. Note that the time step used in step used in the model $(\Lambda t$ sequent time instants for $\left.t_{\text {model }}\right)$; the time Varying $M d$
which the inlet condition
defined, so the total num CFD simulati
opedFixedValueon does not have to
condition interpolates (for eyery $\Delta t_{m}$ only spherical bubbles are defined, so the total number of bubble shapes $N_{\text {shapes }}$ is equal to 1 .

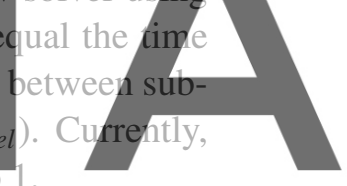

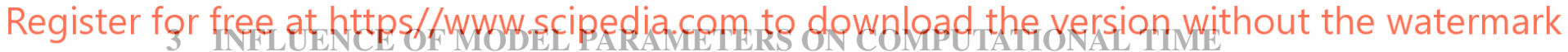

From the discussion above, it is clear that the inlet model defines a transient intet condition with gas bubbles of random size and location based on some input parameters which are set by the user. The most important parameters are: the time step used in the model $\Delta t_{\text {model }}$, the amount of gas $m_{g}$ introduced during a specific time interval $t_{\text {unit }}$ and $t_{\text {unit }}$ itself. The time it takes to complete the inlet model is sensitive to these parameter values, all of which are set by the user. This sensitivity is quantified for a specific case: a 3x5-tube bundle subjected to axial flow.

\subsection{Case description}

The surface mesh of the inlet boundary to be used in this analysis is shown in Figure 1 and contains 3,000 faces. The tube diameter is equal to $9.5 \mathrm{~mm}$ whereas the pitch equals $12.6 \mathrm{~mm}$. The distance between the cell center of an outer cylinder and the nearest outer wall also equals the pitch, leading to a total length of $63 \mathrm{~mm}$ in the $\mathrm{x}$-direction and $37.8 \mathrm{~mm}$ in the y-direction. Slip between the phases is not modelled; both phases enter the domain with velocity $1.5 \mathrm{~m} / \mathrm{s}$. The influence of the following model parameters will be 


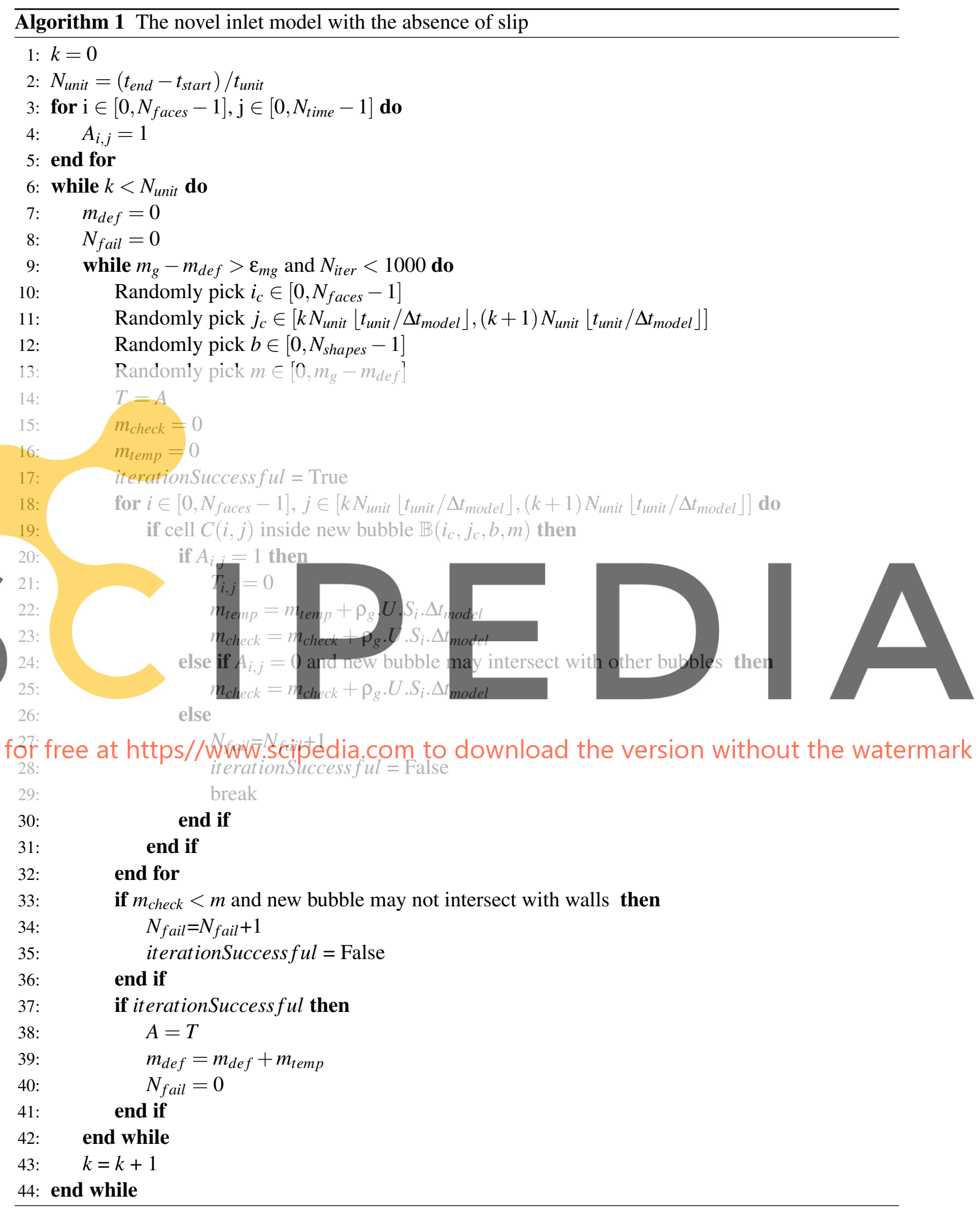




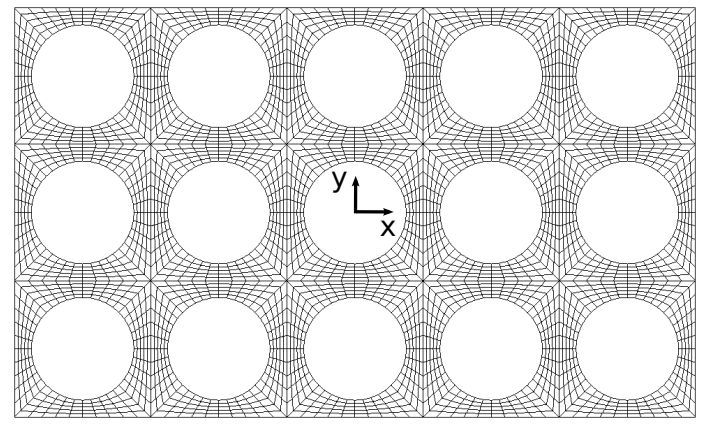

Figure 1: Mesh of the inlet boundary.

investigated: $\Delta t_{\text {model }}, m_{g}$ and $t_{\text {unit }}$. The results of this analysis are discussed in Section 3.2.

\subsection{Computational time required to perform inlet model}

As the model is of stochastic nature, some iterations in Algorithm 1 fail. Running the model multiple times on the same case with the same parameter settings can yield slightly different results in terms of required computational time. Consequently, all datapoints discussed in this section represent the av-

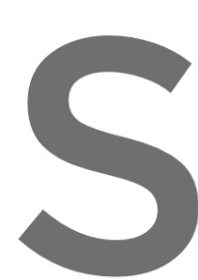
erage duration over three runs, the subsequent CFD sinulation $t$ maximal deviation found erage value. Overall, the average duration for its

Firstly, the influence of $\Delta$

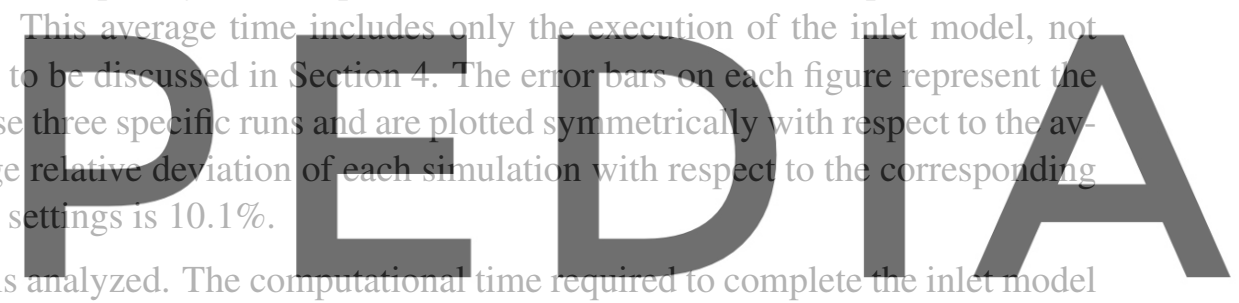
for different values of $\Delta t_{\text {model }}$, is shown in Figure 2. For all simulations reported here, $m_{g}=0.00005 \mathrm{~kg}$

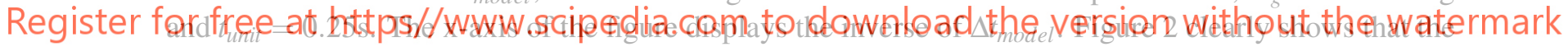
modelling time is inversely proportional of $\Delta t_{\text {model }}$. This is easily explained: doubling $\Delta t_{\text {model }}$ leads to half the number of positions in time to be modelled. Each iteration in Algorithm 1 then also takes half the time to complete.

Secondly, the elapsed time is plotted with respect to the variable $m_{g}$ in Figure 3, for $\Delta t_{\text {model }}=0.001 \mathrm{~s}$ and $t_{\text {unit }}=0.25 \mathrm{~s}$. With increasing $m_{g}$, the computational time for the model increases superlinearly or even exponentially. This is due to the increased number of failed iterations. Clearly, this is a drawback to the current model which could be reduced by implementing a better search algorithm instead of the brute force search that happens during every iteration. This would not alter the superlinear behaviour shown in Figure 3, but would significantly reduce the elapsed time for all datapoints.

Thirdly, Figure 4 shows the modelling time as a function of $t_{\text {unit }}$, for constant $m_{g} / t_{\text {unit }}=0.0002 \mathrm{~kg} / \mathrm{s}$ and $\Delta t_{\text {model }}=0.001 \mathrm{~s}$. The reason for keeping the ratio $m_{g} / t_{\text {unit }}$ constant instead of $m_{g}$ is that the number of failed iterations depends on both parameters and this behaviour has already been discussed when analyzing Figure 3. Probably, a decrease in $t_{\text {unit }}$ (and therefore of available spots to place a new bubble) would result in more failed iterations similar to an increase in $m_{g}$. By keeping the ratio $m_{g} / t_{\text {unit }}$ constant, the 
number of failed iterations relative to the number of successful iterations is hypothesized to be constant for all datapoints in Figure 4, at least on average. This is confirmed by the nearly constant maximal deviation observed in the error bars (except for $t_{\text {unit }}=0.125 \mathrm{~s}$ which has a substantially lower deviation). Also note that the y-scale in Figure 4 spans a significantly smaller range than in the previous figures, showing that the elapsed time does not heavily depend on $t_{\text {unit }}$, as long as the ratio $m_{g} / t_{\text {unit }}$ remains constant. The smaller modelling time found for the lowest value of $t_{\text {unit }}$ could be explained by the fact that, per iteration, only one eighth of the total number of bubble positions needs to be evaluated (as $t_{\text {unit }}$ is one eighth of the total flow time to be simulated). Possibly this allows for more efficient memory management during the run of the Python code.

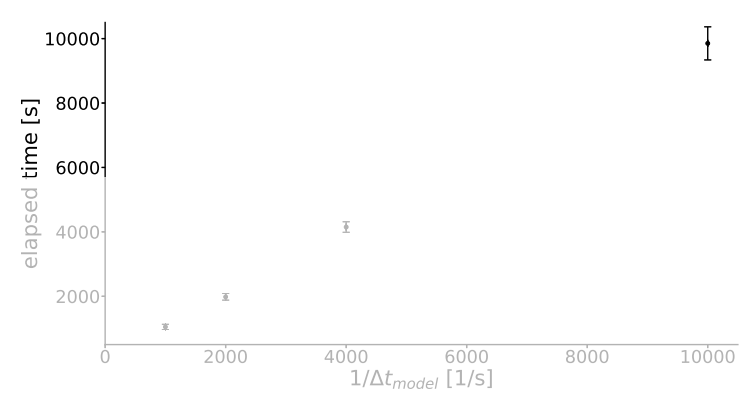

Figure 2: Required time to complete the inlet model as a function of the inverse of the time step $\Delta t_{\text {model }}$. For all datapoints, $m_{g}=0.00005 \mathrm{~kg}$ and $t_{\text {unit }}=0.25 \mathrm{~s}$.
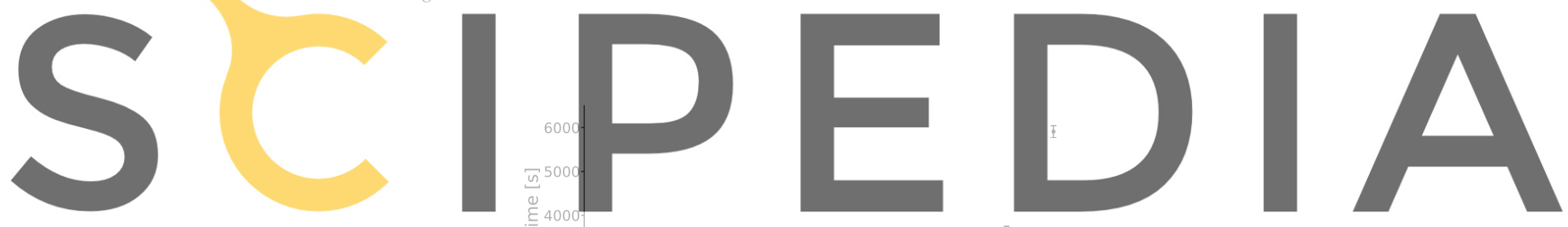

Register for free at https//www

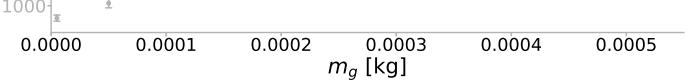

Figure 3: Required time to complete the inlet model as a function of the mass of gas to be introduced $m_{g}$. For all datapoints, $\Delta t_{\text {model }}=0.001 \mathrm{~s}$ and $t_{\text {unit }}=0.25 \mathrm{~s}$. 


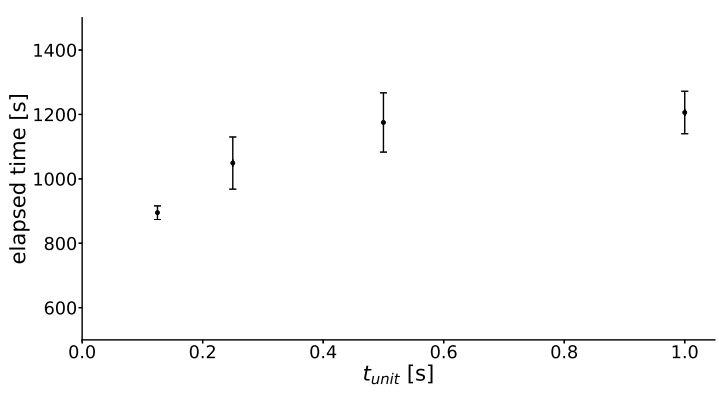

Figure 4: Required time to complete the inlet model as a function of the time interval $t_{\text {unit }}$. For all datapoints, $m_{g} / t_{\text {unit }}=0.0002 \mathrm{~kg} / \mathrm{s}$ and $\Delta t_{\text {model }}=0.001 \mathrm{~s}$.

\section{COMPARISON WITH PRECURSOR DOMAIN SIMULATION}

In this section, the CFD calculation using the inlet model will be compared to simulations using a steadystate inlet with thin air jets. Such simulations require a longer domain (hence, a precursor domain needs to be added in front of the actual computational domain from which the flow data are extracted) because these air jets need to break up and form discrete bubbles in a natural way. While the precursor domains needs to be solved for every subsequent time step, use of the inlet model allows the bubbles to enter the domain directly and therefore to limit the computational effort of the flow simulation to the actual domain of interest. Thei

domain was prolonged by exerted on the tubes.

4.1 Case description
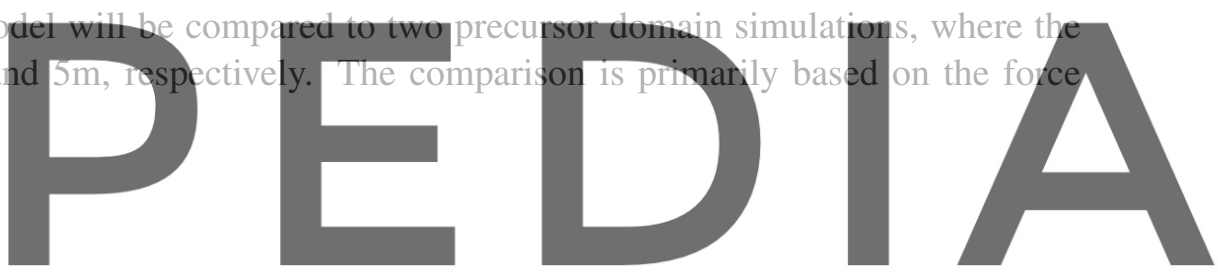

The solver to be used in all CFD calculations is part of the OpenFOAM ${ }^{\circledR} 4.1$ package and is called

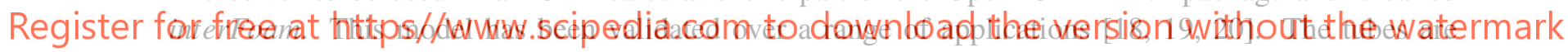
positioned in a square array, as shown in Figure 1. The cross-sectional mesh is the same as used in Section 3.1. The axial length of the domain is $1.52 \mathrm{~m}$, subdivided in 600 equally large divisions. This leads to a total of $1,800,000$ cells in the three-dimensional geometry in case of the simulation using the inlet model. The forces on the cylinders are the result of the integral of pressure and shear stresses in this domain. In case of the precursor simulations, the domain is lengthened with $1 \mathrm{~m}$ or $5 \mathrm{~m}$, respectively. The mesh is constructed in such a way that the axial length of the cells remains (almost) equal for all three cases, hence a total number of 3,000,000 cells is found when the precursor domain is $1 \mathrm{~m}$ long and of 7,764,000 cells is obtained for the $5 \mathrm{~m}$ long precursor domain. The boundary condition at the inlet of the precursor domain is shown in Figure 5 and consists of 6 steady air jets in a continuous water flow. Similar to the inlet model, slip between the phases is not considered at the inlet: the inlet velocity is $1.5 \mathrm{~m} / \mathrm{s}$. 


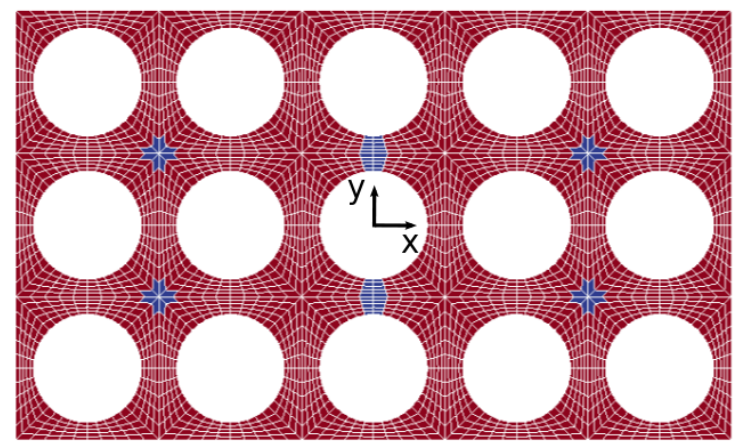

Figure 5: View of the steady boundary condition for $\alpha_{w}$ applied to the entrance of the precursor domain. The blue color indicates the presence of air, while water is shown in red. The mesh edges are shown in white.

\subsection{Results}

The comparison between the simulation using the inlet model and the two precursor domain simulations is mainly based on Figure 6, where the real Fourier transform of the forces exerted on the central tube is plotted. It is clear that a precursor domain of $1 \mathrm{~m}$ does not allow the air jets to develop to large bubbles able to create the forces obtained from the inlet model. On the other hand, if the precursor domain is taken sufficiently long, similar results between the inlet model and the precursor domain simulation can

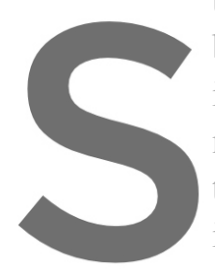
be obtained, even though in the precursor domain noteworthy that the ener the $x$-direction for the $p$ in the $y$-direction. This $y$ the low frequencies do not
are not necessarily of the
y contained in the lowest
would not be the case if
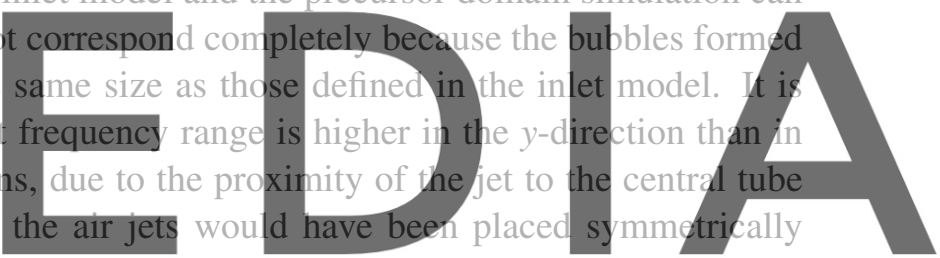
around the central tube. Accordingly, the inlet model does not exhibit a discrepancy between the $x$ - and

Register for free at hthection if the simulation time is sufficiently long. For this particular geometry, no experimental data about the bubble distribution were found, but this analysis shows that the bubble shape and size used in the inlet model need to be chosen with some care.

At least, with the inlet model, this can be set by the user, whereas the precursor domain simulation does not allow any user input in that regard; only if the instability leading to the jet break-up is of physical nature will a precursor simulation be close to the actual flow behaviour.

One of the main objectives during the development of the inlet model, was to make a time-efficient model with an accuracy equal or superior to that of a precursor model, but with less computational cost. The inlet model prior to the CFD simulation took a total of $15,712 \mathrm{~s}$ on a single core (the inlet model only works in serial at the moment) of a 2 x 12-core Intel E5-2680v3 (Haswell-EP @ $2.5 \mathrm{GHz}$ ) node in order to model a transient inlet over a time span of $15 \mathrm{~s}$. The subsequent CFD calculation was performed in parallel, on 120 cores (4 nodes of the same type); in 259,026s, 12.0566s flow time was calculated before the simulation was halted due to exceeding of the maximum available time. Hence, the CFD simulation takes $21,484 \mathrm{~s}$ to calculate $1 \mathrm{~s}$ of flow time. Assuming the computational time for the precursor domain simulation scales with the length of the domain - all other variables remaining the same - this means that a precursor domain of $1 \mathrm{~m}$ and $5 \mathrm{~m}$ long take $35,618 \mathrm{~s}$ and $92,155 \mathrm{~s}$, respectively, to calculate 


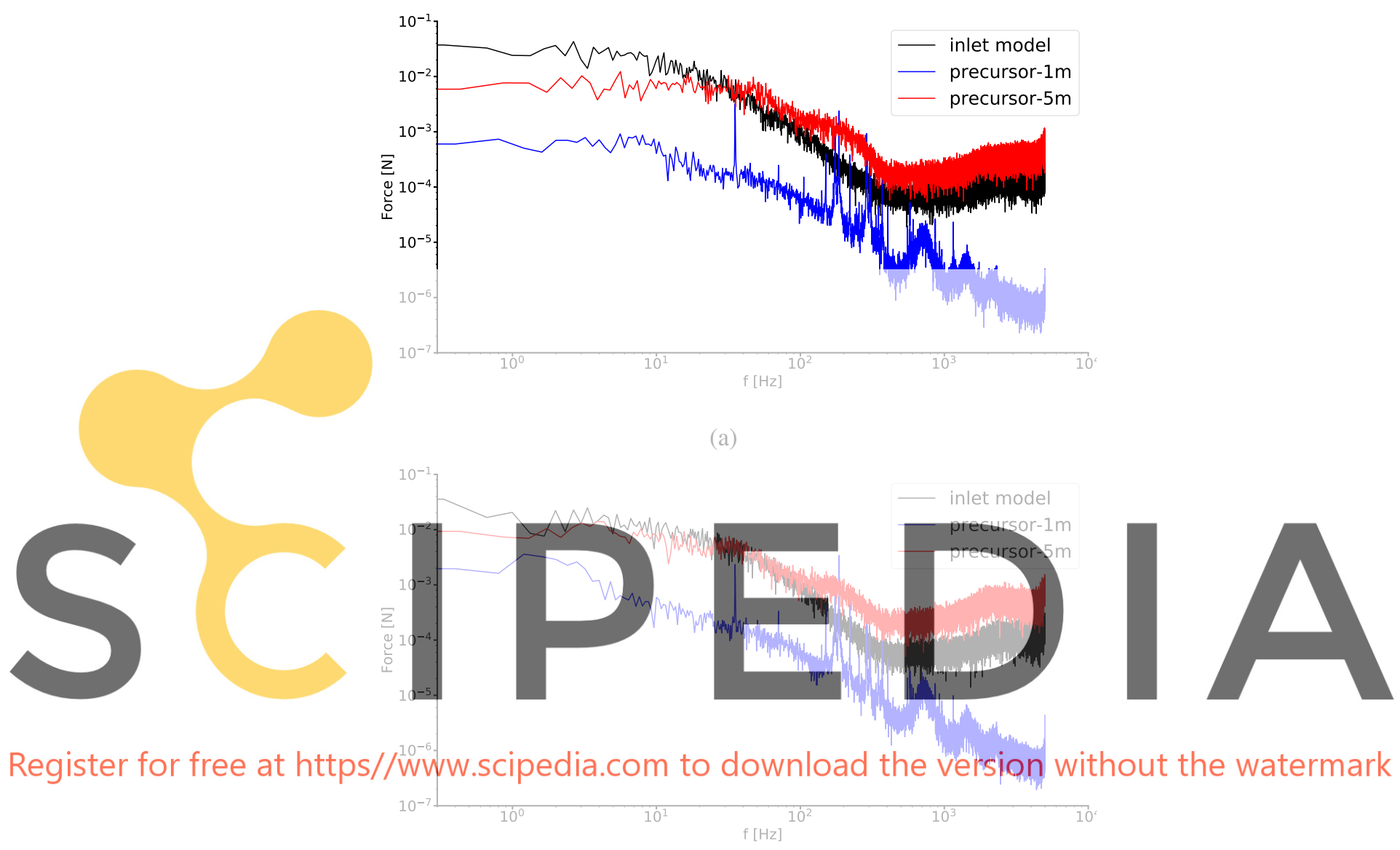

(b)

Figure 6: Real Fourier transform of the force profile on the central cylinder of the tube bundle extracted fom the inlet model simulation (for which the temporal data spans 12s), the simulation with a precursor domain of $1 \mathrm{~m}$ long (15s) and with a precursor domain of $5 \mathrm{~m}$ long (14.1s), respectively. Each time series was split into 4 parts, of which the FFT were calculated individually. The shown result is the average Fourier spectrum of the force (a) in the x-direction and (b) in the y-direction. 
1s of flow time on the same number and type of nodes. Evidently, the actual value of this limit of little importance, but it highlights that the inlet model is more efficient than a precursor domain simulation in cases with a sufficiently long flow time and moderate mesh size and parallellization. Increasing the number of cores in the precursor domain naturally reduces the wall clock time required to perform the precursor domain simulation, which is not the case for the inlet model which operates in serial. However, increasing the number of cores will probably not yield to a linear speed-up of the calculation and requires more computer infrastructure, which is why the inlet model is still a valuable alternative.

\section{CONCLUSION}

A novel inlet model to be combined with the Volume-Of-Fluid method was presented. After describing the algorithm, the influence of the most important modelling parameters was quantified using a $3 \times 5$-tube bundle geometry. It was concluded that the time required to complete the inlet model scales linearly with the inverse of the time step used in the model and is very dependent on the mass of gas to be introduced in the domain. On the other hand, the model parameter $t_{\text {unit }}$ does not affect the computational time to a large extent as long as the average mass flux of gas is kept constant. Subsequently, the force exerted on the central rod in the tube bundle as predicted by the inlet model simulation and two precursor domain simulations were compared. The inlet model and the simulation with the longest precursor domain yield similar results. Additionally, it is less time-consuming for simulations requiring a large domain or a long flow time.

\section{ACKNOWLEDGMENTS}

The authors gratefully acknowledge the funding by the Research Foundation-Flanders (FWO), Belgium, through the Ph.D. fellowship of Laurent De Moerloose. The computational resources (Stevin Supercomputer Infrastructure) and services used in this work were provided by the VSC (Flemish Supercomputer Center), funded by Ghent University, FWO and the Flemish Government department EWI.

\section{REFERENCES}

[1] Ren, Q., Zhou, W., Du, S., Li, Z. and Pan, L. Sub-channel flow regime maps in vertical rod bundles with spacer grids. Int. J. Heat Mass Tran. (2018) 122: 1138-1152.

[2] Liu, H., Pan, L., Hibiki, T., Zhou, W., Ren, Q. and Li, S. Axial development of gas-liquid flow regime maps in a vertical $5 \times 5$ rod bundle with prototypic spacer grids. Nucl. Eng. Des. (2018) 339: $1-10$.

[3] De Ridder, J., Doaré, O., Degroote, J., Van Tichelen, K., Schuurmans, P. and Vierendeels, J. Simulating the fluid forces and fluid-elastic instabilities of a clamped-clamped cylinder in turbulent axial flow. J. Fluids Struct. (2015) 55: 139-154.

[4] De Moerloose, L., Aerts, P., De Ridder, J., Vierendeels, J., and Degroote, J. Numerical investigation of large-scale vortices in an array of cylinders in axial flow. J. Fluids Struct. (2018) 78: 277-298.

[5] Bertocchi, F., Rohde, M. and Kloosterman, J. LDA measurements of coherent flow structures and cross-flow across the gap of a compound channel with two half-rods. Nucl. Eng. Des. (2018) 326: 
$17-30$.

[6] Ricciardi, G. and Boccaccio, E. Measurements of fluid fluctuations around an oscillating nuclear fuel assembly. J. Fluids Struct. (2014) 78: 332-346.

[7] Pettigrew, M. and Taylor, C. Vibration analysis of shell-and-tube heat exchangers: an overview Part 1: flow, damping, fluidelastic instability. J. Fluids Struct. (2003) 18: 469-483.

[8] Gouesbet, G. and Berlemont, A. Eulerian and Lagrangian approaches for predicting the behaviour of discrete particles in turbulent flows. Prog. Energy Combust. Sci. (1999) 25: 133-159.

[9] Nakamura, T., Fujita, K., Kawanishi, K., Yamaguchi, N. and Tsuge, A. Study on the vibrational characteristics of a tube array caused by two-phase flow. Part 1: random vibration. J. Fluids Struct. (1995) 9: 519-538.

[10] Selima, Y., Hassan, M., Mohany, A. and Al-Hamadani, M. A model for fluidelastic instability in tube bundles subjected to two phase flow. Proc. of 9th Int. Symp. Fluid-Structure Interactions, Flow-Sound Interactions, Flow-Induced Vibration \& Noise. (2018): 1-7.

[11] Chesters, A. and Hofman, G. Bubble coalescence in pure liquids. Appl. Sci. Res. (1982) 38: 353361.

[12] Parmar, M., Haselbacher, A. and Balachandar, S. Improved drag correlation for spheres and application to shock-tube experiments. AIAA J. (2010) 48(6): 1273-1276.

[13] Akiki, G., Jackson, T. and Balachandar, S. Pairwise interaction extended point-particle model for a random array of monodisperse spheres. J. Fluid Mech. (2017) 813: 882-928.

[14] Trapp, J. A discrete particle model for bubble-slug two-phase flows. J. Comput. Phys. (1993) 107: 367-377.

[15] Hirt, C. and Nichols, B. Volume of Fluid (VOF) Method for the dynamics of free boundaries. $J$. Comput. Phys. (1981) 39: 201-225.

[16] Venkateswararao, P., Semiat, R. and Dukler, A. Flow pattern transition for gas-liquid flow in a vertical rod bundle. Int. J. Multiphase Flow (1982) 8(5): 509-524.

[17] De Moerloose, L. and Degroote, J. A study of the vibration of a horizontal U-bend subjected to an internal upwards flowing airwater mixture. J. Fluids Struct. (2020) 93: 102883.

[18] Deshpande, S., Anumolu, L. and Trujillo, M. Evaluating the performance of the two-phase flow solver interFoam. Comput. Sci. Discovery (2012) 5: 014016.

[19] Shuard, A., Mahmud, H. and King, A. Comparison of two-phase pipe flow in OpenFOAM with a mechanistic model. IOP Conf. Ser: Mater. Sci. Eng. (2016) 121: 012018.

[20] Larsen, B., Fuhrman, D. and Roenby, J. Performance of interFoam on the simulation of progressive waves. Coastal Eng. J. (2019) 61(3): 380-400. 